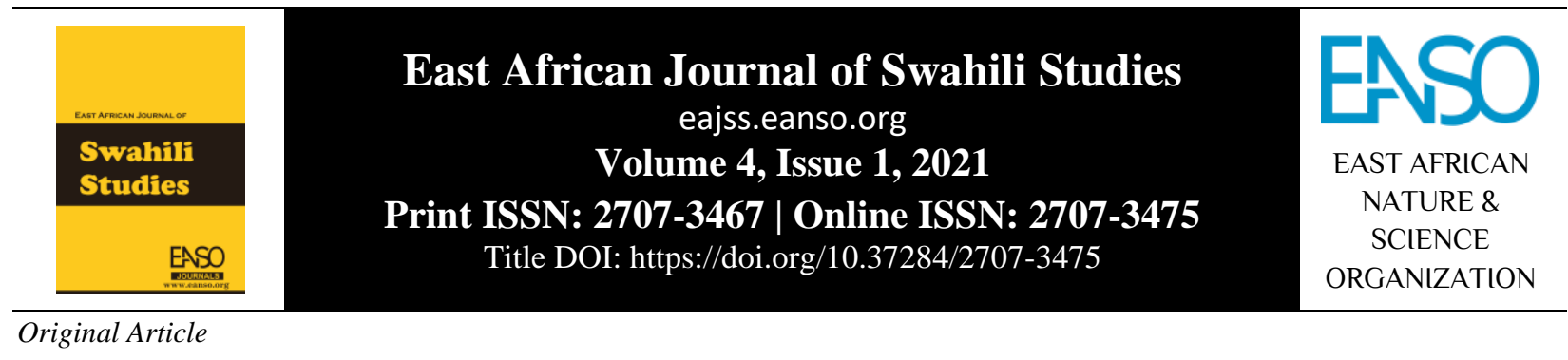

\title{
Ishara Bwege Katika Diwani ya Kichomi.
}

\section{Chibayi Jason Poyi ${ }^{1}$}

${ }^{1}$ Chuo Kikuu cha Laikipia, S.L.P 1100 - 20300. Nyahururu, Kenya.

*Barua pepe ya mawasiliano: jasonchibayi@gmail.com

DOI ya Nakala: https://doi.org/10.37284/eajss.4.1.446

\section{Tarehe ya Uchapishaji: IKISIRI}

26 Ocktoba 2021 Wasomi na watafiti wanadai kwamba ishara ni sehemu muhimu ya mtindo. Wanakubaliana kwamba ishara ni kigezo muhimu katika kufasiri maisha

Istilahi Muhimu: bwege. Ishara huonyesha hali halisi ya maisha bwege ya binadamu. Kwa hivyo, makala haya yananuia kuhakiki ishara zilizotumika kuashiria ubwege Ishara, wa maisha katika diwani ya Kichomi. Utafiti huu uliongozwa na nadharia ya Ubwege, Mtindo iliyoasisiwa na Leech ambayo itasaidia katika kuhakiki ishara Mtindo, zilizotumika kuibua ubwege wa maisha katika mashairi teule ya diwani ya Sawiri. Kichomi. Ishara zilifafanuliwa na kuwasilishwa kwa njia ya maelezo yaliyoegemezwa kwenye mihimili teule ya nadharia ya Mtindo. Uchanganuzi wa ishara uliongozwa na mbinu ya uhakiki matini. Katika diwani ya Kichomi, Kezilahabi anaangazia ubwege kwa njia ya kifalsafa na usanii wa hali ya juu kwa kutumia ishara maalumu. Mtafiti alihakiki matumizi ya ishara kwa namna ambavyo yalitumika kuangazia ubwege. Makala haya yatawafaa waandishi wa fasihi hasa ushairi kwani yatawawezesha kuelewa mbinu mbalimbali za kuandika matini zenye mvuto wa kisanaa ili kuwasilishia jamii ujumbe kwa ubunifu mkubwa.

\section{APA CITATION}

Poyi, C. J. (2021). Ishara Bwege Katika Diwani ya Kichomi. East African Journal of Swahili Studies, 4(1), 12-29. https://doi.org/10.37284/eajss.4.1.446.

\section{CHICAGO CITATION}

Poyi, Chibayi Jason. 2021. "Ishara Bwege Katika Diwani ya Kichomi”. East African Journal of Swahili Studies 4 (1), 12-29. https://doi.org/10.37284/eajss.4.1.446.

\section{HARVARD CITATION}

Poyi, C. J. (2021) "Ishara Bwege Katika Diwani ya Kichomi”, East African Journal of Swahili Studies, 4(1), pp. 12-29. doi: 10.37284/eajss.4.1.446.

\section{IEEE CITATION}

C. J. Poyi, “Ishara Bwege Katika Diwani ya Kichomi”, EAJSS, vol. 4, no. 1, pp. 12-29, Oct. 2021.

12 This work is licensed under a Creative Commons Attribution 4.0 International License. 


\section{MLA CITATION}

Poyi, Chibayi Jason. "Halafu ya Ubunifu wa Kiafrika: Mtanziko Rejeleshi wa Tawala za Kiafrika". East African Journal of Swahili Studies, Vol. 4, no. 1, Oct. 2021, pp. 12-29, doi:10.37284/eajss.4.1.446.

\section{UTANGULIZI}

Uwasilishaji ujumbe katika mashairi ya diwani ya Kichomi umefanywa kwa njia sahili ya ishara ambazo zimesheheni falsafa nzito ya maisha ambayo itakuwa dhahiri tu kwa mwenye kuzitazama kwa jicho la ndani. Euphrase Kezilahabi anaona mtindo wa lugha za ishara kama sehemu ya lugha ya kawaida ambayo imetawala mazungumzo ya binadamu wakati anapotangamana na binadamu wenzake duniani. Kwa hivyo, matumizi ya lugha ya aina hii katika mashairi yanafaa kuchunguzwa ili kubaini namna ilivyotumika katika uwasilishaji wa suala la ubwege wa maisha katika ushairi huu wa kisasa.

\section{Dhana ya Ubwege}

Dhana ya ubwege katika fasihi ina fasili nyingi. Kwa mujibu wa Edeh (2019), ubwege hudhihirika kama maudhui ya udhanaishi yanayosisitiza kukosekana kwa maana na ufahamu maisha ya binadamu. Naye Stein (2019) anasema kwamba Eugene Ionesco anaufasili ubwege kuwa kama hali tu "isiyo na kusudi, iliyotokana na kudorora kwa misingi ya kidini, metafizikia na ontolojia na kumsawiri binadamu kama ambaye amepotea." Anaendelea na kusema kwamba "vitendo vyote vya wanadamu vinakuwa visivyo na maana na vya kipuuzi." Kwa upande wake, Kipacha (2019) anauona ubwege kama mtazamo wa kidhanaishi unaopinga kwa jinsi yoyote ile kuwepo kwa maana ya maisha. Anashikilia kuwa juhudi za binadamu za kujitungia maana hiyo ni upuzi mtupu kwa sababu kunakinzana na hali halisi ya kukosekana kwa maana.

Camus (2005) anafasili ubwege kama wanadamu kutokuwa na uwezo wa kuyafanya maisha yao yawe na maana. Anadai kwamba, ubwege ni zao la mkanganyiko unaotokea kati ya hamu ya maana ya kuwako na kutoweza kupata maana yoyote. Naye Esslin (2001) anasema kwamba, ubwege ni kile ambacho kimekosa nia, kimetengwa kutoka kwa imani na tabia za kiungu na mizizi ya kiakili na mawazo. Esslin (2001) anashikilia kuwa ubwege unadadisi hali ya binadamu kuendelea kuishi ilhali maisha hayana maana tena. Akimnukulu Camus (2005), Bennette (2011) anasema kuwa ulimwengu ambao ghafla umehiniwa udanganyifu na nuru, binadamu huhisi kuwa mgeni. Hujiona akiwa kwenye uhamisho usioweza kuepukika kwa sababu amenyimwa kumbukumbu za dunia iliyopotea, na hana tumaini la ulimwengu wa ahadi anaousubiri. Talaka hii kati ya binadamu na maisha yake, mwigizaji na mpangilio wake kweli huibusha hisia ya upuuzi. Kwa tafsiri hii, Camus anaibua taswira kamili ya hisi za binadamu aliyechanganyikiwa, kukata tamaa na kukosa tumaini inayoashiria mzozo wa ndani kati ya mwanadamu, uwepo wake na kusudi lake.

Kwa mujibu wa Mumme (2016), mjadala wa ubwege kama suala maalum la kifalsafa lina misingi yake kwenye tungo zake Søren Kierkegaard. Yeye alitumia neno hilo kuonyesha mzozo wa kiimani na kirazini uliyoikumba dini ya Wakristo. Hata hivyo, Mumme (2016) anakaulisha kwamba ni Camus aliyendeleza dhana ya ubwege kupitia kwa vitabu vyake vya The Myth of Sisyphus (2005), The Outsider (1961), The Plague (2009), The Rebel (1991) na Resistance, Rebellion, and Death (1961). Fasili ya Camus (keshatajwa), inayasawiri maisha kama yasiyokuwa na maana yoyote. Licha ya maisha kuwa ovyo na kukosa maana, Camus anadai kwamba, ni sharti tuyastahimili na kuibuka na malengo ya kishujaa kama Sisyphus kwenye mchezo wa The Myth of Sisyphus (Mumme, 2016). Bennett (2011) na Tarantino na Caruso (2009) wanaainisha sifa bainifu za ubwege wa Camus. Kwao, ubwege huyasawiri maisha kama yasiyokuwa na maana yoyote. Wao huona juhudi za binadamu kujitungia maana huwa za kipuuzi. Wanausawiri ubwege ambao unamwonyesha binadamu kama ambaye amekubali kuishi na machungu. Wanamulika juhudi za binadamu za kutafuta furaha kama bure na ovyo. Waaidha, huwasilisha malalamishi machungu kuhusu binadamu aliyekatishwa tamaa na maisha ya sasa. Kadhalika ubwege huisawiri jamii ambayo badala ya kujitolea kidini, imekweza maazimio ya mtu binafsi dhidi ya kila kitu. Vilevile ubwege unaonyesha namna silisili zilizounganisha familia na kuyajaza maisha na hisia za maana zilinavyokosa athari yoyote kwenye maisha ya sasa. Isitoshe, 
ubwege husawiri namna wanaojaribu kutimiza malengo yao kibinafsi kupitia kwa mapenzi, maisha yao yanavyosalia bila hisia, na namna wengi wao wanavyong'ang'ana na huzuni na majonzi. Hata wale wanaopata mapenzi, huwa ni ya muda mfupi na yanayoandamana na mauaji au hisia ya udanganyifu. Kwa mujibu wa Camus (2005), uzee huandamana na aibu ya mwili kimaumbile, kutelekezwa na upweke unaowafanya wengi kuafadhalisha kufariki.

\section{Dhana ya Ishara}

Frye na wenzake (1997) wanasema kwamba, "ishara ni kiashirii na kile inachosimamia ni kiashiriwa." Kwa mtazamo wao, ishara huelekea kuwakilisha kidhahania, maana na hali ya kile kinachoashiriwa. Naye Meyer (2002) anadai kuwa, "maana za ishara zimekitwa katika mpangilio wa matini na kwa hivyo zinahimiliwa na miktadha ya matumizi yake. Miktadha hii inaweza kuwa ya kidini, kisiasa, kijamii au ya kiuchumi." Perrine (2014) anasema, "ishara ni kitu kilicho na maana zaidi ya kitu hicho. Yaani, ishara ni kitu, mtu, hali, tukio au kitu chochote kile kilicho na maana ya kawaida lakini kinaashiria au kinawakilisha maana nyingine zaidi." Kutokana na mawazo ya Perrine (2014), "ishara ni kama kitu cha thamani chenye rangi nyingi kinachoweza kudhihirisha rangi mbalimbali kinapowekwa kwenye mwangaza." Kwa mujibu wa fasili hii, ni dhahiri ishara sio tu kitu bali pia hurejelea tukio, hali au mtu anayeweza kutumiwa kudokeza maana kiishara (Mukhata, 2015). Snowden (2006) anasema kuwa, ishara huhusisha vitu hai, visivyo hai na vitu dhahania. Kwa mujibu wa Snowden (keshatajwa), kitu, hali au tukio linalotumiwa kiishara katika matini za kifasihi huwa na maana iliyo zaidi ya maana ya kawaida na inayohusiana na kiashirii. Kwa maana hii basi nimeiangalia ishara kama kitu, hali, tukio, neno, mtu au mandhari yanayowakilisha maana ya ziada ya kiashirii na yenye mahusiano na kiashirii hicho. Maana hii ndiyo nimeichukulia kama fasili ya ishara kwenye makala hii.

Wahakiki wa tungo mbalimbali za Kezilahabi wameziainisha matini zake kuwa za ubunifu wa kiwango cha juu, ambazo zimewasilishwa kwa lugha rahisi lakini yenye mafumbo, ishara na yenye misemo iliyosheheni falsafa ya kidhanaifu (Kahigi, 2020; Kipacha, 2019, 2017; Wamitila 1998;
Gromov 1998; Diegner 2002, 2005; Khamis 2003; Rettova 2004; Lanfranchi 2012; Gaudioso, 2014, 2015). Kahigi (2020) anachambua sitiari katika diwani ya Kichomi ya Euphrase Kezilahabi. Mashairi aliyoyachunguza yalishughulikia maudhui tatu ya kijumla: "maana ya maisha, udhalimu wa kikoloni, na hali ya sasa ya Afrika" (Kahigi, 2020).

Katika utafiti wake, Kahigi alionyesha kwamba sitiari ndio mbinu ya kimsingi ya mshairi katika kuwasilisha maana na mtazamo wake kwenye maudhui anayoyachimuza. Utafiti wake Kahigi umemsawiri Kezilahabi kama mtunzi aliyetumia mbinu ya sitiari kama nyenzo muhimu kabisa ya kisanaa katika kuyasawiri mawazo na hisia zake kuhusu maana ya maisha, madhara ya ukoloni, na hali halisi ya Afrika kwenye mashairi yake. Aidha, Kahigi anaaisha ishara, mbazi na isitiara kama tamathali ambazo zina msingi kwenye sitiara. Utafiti wake ni muhimu kwa utafiti huu wa sasa kwa sababu umechunguza namna sitiari ilivyochangia katika ukuzaji wa maudhui na hususan maana ya maisha. Aidha, uhakiki wa Kahigi wa sitiari ni mchango mkubwa kwa makala hii. Uhakiki huu vilevile umehakiki ishara kwenye diwani ya Kichomi sawa na utafiti wa Kahigi. Isitoshe, uchambuzi wa sitiari umehusisha mkabala wa kisemantiki wa nadharia ya mtindo na hivyo basi kumjengea mtafiti msingi imara wa nadharia ya umitindo ambayo imetumika kwenye makala hii

Euphrase Kezilahabi yupo miongoni mwa pote la wabidaa wa ushairi ulimwenguni. Madai anayothibitisha Kipacha (2019) kupitia kwenye makala yake, "Ushairi msemele na ubidaa wa Euphrase Kezilahabi katika Dhifa." Kipacha anasema kwamba Kezilahabi amevichipuza vipengele msemele ili kuwarai wasomaji wake wayakumbatie mabadiliko ya kimawazo na kimaudhui kwenye ushairi wake. Kauli ambayo Gaudioso (2015) anaiunga mkono anapodai kwamba Kezilahabi anawahimiza watu wayakumbatie na kuyakubali mabadiliko ilhali yeye mwenyewe ndiye anayezalisha mabadiliko hayo.

Kipacha (2019) anamsawiri Kezilahabi akitumia zana ya ubezi kuipindua misemo, hekima au methali zilizozoeleka katika jamii ya Waswahili ili kujenga fikra mpya au kuzibidaa kimtazamo. Kupitia kwa zana za kimsemele, Kipacha anasema kwamba Kezilahabi ameweza kuufunua na kuuweka wazi 
msimamo na muonoulimwengu. Uthubutu ambao msomi huyu anasema kwamba umempatia Kezilahabi kilemba cha ubidaa duniani. Utafiti wa Kipacha ni muhimu kwa utafiti wa sasa kwa sababu umehakiki kipengele msemele ambacho ni sehemu ya ubidaa wa kiishara na kimaudhui ambao Kezilahabi alidhamiria kuibua katika ushairi wa Kiswahili. Hivyo uhakiki huu ni hatua zaidi ya utafiti wa Kipacha ijapokuwa umechunguza namna ishara ambazo ni miongoni mwa za zana za ubidaa wa Kezilahabi, zinavyoibusha suala ubwege kwenye diwani ya Kichomi tofauti na Kipacha aliyehakiki diwani ya Dhifa.

Kwa kuchomoza ishara za kiuchuro kwa wingi kwenye utunzi wake mbalimbali, Kipacha (2017) anadai kwamba, Euphrase Kezilahabi amefanikiwa kuibua fikra mpya inayokinzana na ile fikra iliyozoeleka ya mfumo wa thamani na kuadili iliyopo kwenye jamii yake. Kwa mujibu wa Kipacha (2017), Kezilahabi anapinga maana na fasiri ya jumla ya ishara za kiuchuro katika jamii yake kwa kuicheka jumuia nzima inayozishabikia. Anasisitiza kwamba Kezilahabi amevunja dhana za msingi za ishara za uchuro zinazotawala katika jamii yake na ulimwengu kwa ujumla. Kipacha (2017) anadai kwamba kupitia ishara hizi za uchuro, Kezilahabi anawasawiri binadamu ambao wanahofia anguko lao. Anaendelea kusema kwamba Kezilahabi anaweka jinsi wahusika wake wanavyowakilisha viumbe wanaopambana na harakati za kuishi, wenye silika na utashi wa kuishi na wapo katika misuguano na nguvu za kimaumbile na za kijamii ambazo huwakabili kila uchao.

Isitoshe, anadai kuwa kwa vile maumbile hayamchukulii mwanadamu na viumbe vilivyomo kama viumbe muhimu vinavyostahiki kuonewa huruma, Kezilahabi anaenda mbali zaidi katika kazi na kuonyesha udhaifu wake kwa kutumia lugha nyepesi lakini iliyosheheni ishara zenye hisia kali kwa wasomaji. Utafiti wake Kipacha (2017) unahoji mfumo wa ishara za uchuro kwenye tungo za Euphrase ili kuutandua utandu na kukarabati fikra zilizofumbatwa na mfumo wa fikra hafidhina wa jamii yake. Licha ya kuwa Kipacha amehakiki ishara za uchuro kwenye riwaya zake Kezilahabi, utafiti huu umenufaika kutokana nao kinadharia na kiishara. Kwanza, amehimili data yake kwa misingi ya nadharia ya umitindo ambayo imetumika kwenye makala hii. Pili, ameujengea makala hii msingi wa kuhakiki matumizi ya ishara katika kuibusha suala la ubwege kwenye mashairi ya diwani teule.

Kwa maoni yake Mulokozi (2017), Kezilahabi anatumia lugha rahisi ya mazungumzo, lakini iliyosheheni tamathali hasa, ishara, sitiari, taswira na udokezi. Anasisitiza kuwa anaandika mashairi magumu lakini kwa lugha rahisi. Anasema kwamba Kezilahabi anatumia jazanda na ishara kwa sababu ndio moyo wa ushairi wa jadi wa Kiafrika. Kwa mujibu wa Mulokozi (2017), Kezilahabi anatumia ishara za vijijini, majini (mamba, mashua), mimea na mbegu, visasili na visakale, ngoma na duara kuumba maudhui yake. Maoni ya Mulokozi ni mwafaka kwa makala hii kwa sababu yameainisha vipengele vya kimtindo hususan ishara anazotumia Kezilahabi katika kusana itikadi ya ubwege katika mashairi yake na hasa diwani ya Kichomi iliyohakikiwa.

Katika kuufunua mtindo wa utunzi wa Kezilahabi, Wamitila (1998), anamsawiri Kezilahabi kama mtunzi ambaye "yupo kwenye kiwango cha juu cha waandishi wanaojishughulisha na uumbaji tata wa picha na ishara kwenye fasihi ya Kiswahili." Wamitila anadai kwamba ni muhali kwa wasomaji wa kijuujuu kuziibusha na kuzifasiri kwa undani mbinu za utunzi za Kezilahabi. Maoni haya ya Wamitila ni muhimu kwa utafiti wa sasa kwa sababu yamechimuza mbinu ya ishara anayotumia Kezilahabi katika utunzi wake. Utafiti huu umehakiki matumizi haya ya ishara kwenye mashairi ya diwani ya Kichomi ili kufasiri namna zinavyoashiria suala la ubwege katika diwani teule. Hata hivyo, maoni yake Wamitila yanatofautiana na utafiti wa sasa kwa sababu hayakuwa na msingi wa nadharia ya Umitindo ambayo imehimili utafiti huu.

Akihakiki iwapo Nagona na Mzingile ni riwaya, masimulizi au mafumbo, Gromov (1998) anadai kwamba Kezilahabi ametumia sana mbinu ya kimasimulizi hasa visasili vya watu wa jamii yake ya Wakerewe pamoja na visasili vya kilimwengu. Matumizi ya visasili hivi yanaibua matumizi ya wanyama, wadudu, ndege, hali na hisi ambayo ikifasiriwa vyema inaweza kuwa kiashirii cha dhana fulani katika maisha ya binadamu. Kupitia kwa matumizi ya ishara hizi , Gromov anadai kwamba, Kezilahabi ametandua na kukarabati fikira juu ya maisha iliyofumbatwa katika mfumo wa jamii. Fumbo ambalo Rettova (2004) anasema ufumbuzi 
wake kupitia kwa kazi bunilifu za Kezilahabi litasaidia kuibusha mwonoulimwengu na itikadi ya jamii kuhusu maisha. Hata hivyo, utafiti wa Gromov (1998) unatofautina na utafiti wa sasa kwa namna mbili yaani, kimatini na kimwelekeo: kimatini, Gromov amehakiki novela mbili za Euphrase Kezilahabi tofauti na utafiti wa sasa ambao umechunguza mashairi ya diwani ya Kichomi. Kimwelekeo ni kwamba, Gromov alinuia kuthibitisha kama novela hizi ni riwaya, masimulizi au fumbo tofauti na utafiti wa sasa uliohakiki matumizi ya ishara kwenye uashiriaji wa suala la ubwege katika diwani teule ya Kichomi. Hata hivyo mchango wake hauwezi kupuuzwa.

Ishara za mahubiri zimehakikiwa na Mezger (2002) aliyechunguza athari za ishara za kikatoliki katika riwaya ya Mzingile ya Euphrase Kezilahabi. Kwenye makala haya, Mezger (2002) anadai kwamba kupitia kwa ishara hizi za mahubiri, Kezilahabi ameudhihaki mfumo mzima wa thamani ya dini. Mezger anabaini kwamba, ishara hizi za mahubiri zinaelekea kuashiria kuwa dini haina lolote la kumfaa binadamu na kwamba Mungu ni kiumbe cha binadamu pweke. Utafiti huu wa Mezger ni muhimu kwa utafiti wa sasa kwa sababu unahakiki kipengele cha mtindo wa ishara ambacho ni sehemu ya madhumuni yaliyohakikiwa kwenye utafiti huu. Hata hivyo, utafiti wake Mezger (2002) umetofautiana na utafiti wa sasa kimatini na kinadharia. Utafiti huu umehakiki namna ishara zinavyoibusha suala la ubwege kwenye diwani ya Kichomi kwa kuhimili data yake kwenye mihimili ya nadharia ya umitindo tofauti na Mezger aliyechunguza athari za ishara za kikatoliki katika riwaya ya Mzingile.

Osore (2011) na Diegner (2002) wamehakiki ishara mbalimbali katika riwaya ya Rosa Mistika. Diegner (2002) amechunguza ishara za kiistiara kwenye riwaya teule. Kwenye utafiti wake, Diegner anabaisha kwamba mwewe, kuku na vifaranga ni viashirii mfumo dume (mwewe) unavyokandamiza wanawake na wasichana (kuku na vifaranga). Utafiti wake Diegner unaonyesha matumizi ya ishara kwenye riwaya teule kama yanayoibusha dhana ya ukandamizaji. Kwa upande wake Osore (2011), anachunguza ishara za ndoto kwenye riwaya ya Rosa Mistika ya Euphrase Kezilahabi, kama mbinu ya uajinabishaji. Osore (2011) anatambua kwamba ndoto zinaibua ishara muhimu kwenye maisha ya binadamu. Katika utafiti huo, Osore anamsawiri Kezilahabi kama mtunzi anayezitumia ndoto kama nyenzo kuu ya kuweka jukwaani ukweli wa maisha uliofichika. Anazionyesha wazi ndoto kama sehemu ya ishara za kinajimu kwenye maisha ya binadamu. Ijapokuwa, Osore anahakiki ishara ya ndoto kwenye riwaya ya Rosa Mistika, utafiti wake unautajirisha utafiti wa sasa kwa kuujengea msingi mwafaka wa kuhakiki ishara ya ndoto kwenye mashairi ya diwani ya Kichomi.

Akihakiki uhamishaji na kuandika upya uhuru katika mashairi ya Kezilahabi, Gaudioso (2015) anachunguza namna athari za wanafalsafa wa Kijerumani Martin Heidegger na Friedrich Nietzsche zinavyojitokeza katika mashairi ya Kezilahabi. Gaudioso (2015) anabainisha kwamba wanafalsafa hawa walimwathiri Kezilahabi sio tu katika maudhui na mtindo wa kuandika, bali pia katika fikira za ukombozi na uhuru wa Mwafrika. Gaudioso (2015) anadai kwamba, athari ya wanafalsafa hawa ilimjengea Kezilahabi msingi wa kuhamisha mtindo na kuandika upya mawazo yao kwa kutumia ishara na sitiari kwenye kazi zake. Kwenye makala hii, Gaudioso (2015) anahakiki mashairi ya Kezilahabi kwa kuzingatia kigezo cha uhuru wa falsafa ya Nietzsche ili kuonyesha uhusiano na mlingano wacho na wa dhana fulani za falsafa ya Kezilahabi. Gaudioso alijikita katika matumizi ya ishara na istiari katika makala zake ambazo anazionyesha kama kigezo muhimu cha falsafa ya Kezilahabi na ile ya Nietzsche katika kuwasilisha ujumbe. Anadai kwamba Kezilahabi anatumia sana sitiari na ishara kwa sababu inafanya watu kuuona na kuuhisi ushairi wake. Japokuwa haya yalikuwa makala linganishi ya falsafa ya Nietzsche katika mashairi ya Kezilahabi, yanaibua matumizi ya ishara katika mashairi ya Kezilahabi ambayo mtafiti ameyalenga kwenye utafiti huu ili kuonyesha namna matumizi yake yamekuza suala la ubwege. Kimtazamo, utafiti huu wa sasa unatofautiana na utafiti wa Gaudioso kwa sababu, umeonyesha matumizi ya ishara katika kuibua suala ubwege kwa kutumia nadharia ya umitindo.

Mukhata (2015) anachanganua namna ishara na uashiriaji zilivyo nyenzo ya mtindo ya kuwasilishia maana na kujenga uhusika na maudhui kwenye riwaya ya Nyuso za Mwanamke (2010) ya Said Ahmed Mohamed. Anaangazia mchango wa ishara 
bia, ishara za kaida na ishara za kifasihi katika kuwasilisha maana na imani ya jamii katika riwaya teule. Katika utafiti wake, Mukhata anabaini kwamba uashiriaji umefaulu kuibusha maana ainati za kifasihi, kuendeleza maudhui na kuwasawiri wahusika. Anaonyesha namna ishara zinavyokuza maudhui ya mapenzi, itikadi na utamaduni, dhuluma dhidi ya wanawake, umaskini, uharibifu wa mazingira na haki za wanawake kwenye matini teule.

Aidha, Mukhata anabainisha kuwa wahusika wametumiwa kiishara ili kuusawiri uhusika kama wa kipekee kwenye riwaya teule. Mukhata anahitimisha makala yake kwa kudai kwamba mwandishi alifaulu kutumia ishara kuwasilisha maana, kukuza maudhui na kuwakilisha itikadi au utamaduni wa jamii husika. Utafiti wa Mukhata ni muhimu kwa utafiti wa sasa kwa sababu umehakiki ishara na uashiriaji na hivyo basi kuujengea utafiti huu msingi wa kuhakiki mtindo wa kiishara katika diwani ya Kichomi. Hata hivyo, utafiti wa sasa umetofautiana na utafiti wa Mukhata kwa jinsi mbili: kwanza alihakiki riwaya ilhali utafiti huu umechunguza diwani ya mashairi ya Kichomi. Pili ni kwamba, Mukhata ameangazia namna ishara na uashiriaji zinavyoibua maana, kukuza wahusika na kuchimuza maudhui ilhali utafiti wa sasa unaangazia tu namna ishara zinavyoashiria suala la ubwege katika diwani ya Kichomi.

Katika makala yake, "kuwako na wakati: mipaka ya matumizi ya lugha katika mashairi ya Kezilahabi," Gaudioso (2014) alieleza nafasi ya matumizi lugha kwenye diwani za Kezilahabi. Alifunua pia mgogoro baina ya lugha ya ushairi (hasa fasihi andishi) na jamii na baina ya maandiko na maumbile-ulimwengu. Kadhalika Gaudioso (2014) aliangazia kelele iliyochangiwa kwa mgogoro huo, na lugha ya kimya ambayo imekuwa lugha ya utopia inayochangia tafiti nzito sana za Kezilahabi. Gaudioso (2014) anaonyesha namna Kezilahabi anavyousawiri mgogoro huo katika mashairi kama ishara tosha kwamba ulisambaa hadi Tanzania. Kadhalika, katika utangulizi wa utafiti huu, Gaudioso (2014) anasawiri jinsi Tanzania ilivyokuwa na changamoto mbalimbali za lugha ya fasihi na ambayo Kezilahabi anaitatua kwenye fasihi yenyewe, kifalsafa na kishairi, na kuanzisha kurasa mpya kwa fasihi ulimwenguni. Gaudioso (2014) anafafanua falsafa ya Kezilahabi kilinganishi na kiutafsiri. Anamlinganisha Kezilahabi na wanafalsafa wa Kijerumani Martin Heidegger na Friederich Nietzsche, ili kuonyesha namna vipengele vya ishara, sitiari, na mafumbo vya Kezilahabi vinavyoingiliana na vile vya Heidegger na Nietzsche.

Ijapokuwa makala ya Gaudioso (2014) yaliangazia falsafa ya Kezilahabi kilinganishi na kiutafsiri, yalibua suala la mgogoro wa lugha ya kawaida iliyopaswa kuwa ya kishairi, ya Kezilahabi ikiwemo, na kuonyesha ari yao kutumia lugha ya mawasiliano kawaida. Utafiti huu umehakiki matumizi ya lugha ishara ambayo ni sehemu ya lugha ya kawaida anayoizungumzia Gaudioso katika mashairi ya Kezilahabi. Tofauti na Gaudioso (2014) aliyeyalinganisha matumizi ya lugha ya Kezilahabi kiubwege na ile ya Heidegger na Nietzsche, utafiti huu ulilenga kuonyesha namna matumizi ya ishara yalivyoibusha suala la ubwege katika mashairi ya diwani teule ya Kichomi..

\section{Misingi ya Kinadharia}

Nadharia iliyotumika kuhakiki ishara katika mashairi ya Kichomi ni ile ya umitindo. Nadharia hii ni muhimu katika kuchunguza maudhui ya msanii pamoja na matumizi ya lugha ambayo ndiyo huibusha maudhui hayo. Nadharia hii inashikilia kwamba kazi ya fasihi haiwezi kueleweka bila lugha. Kwayo, jinsi mtunzi atumiavyo lugha husaidia kutoa ujumbe wake kwa wasomaji au wasikilizaji. Hivyo, lugha huwa ndicho chombo muhimu cha mawasiliano katika jamii zote za wanadamu kama asemavyo Leech (1969:4) kwamba:

\section{Kazi ya fasihi haiwezi kueleweka vizuri bila kuwa na ufahamu}

mzuri wa lugha ambayo hutumiwa kuieleza. Ni muhimu kuelewa

uhusiano ulioko kati ya lugha ya ushairi na ujumbe wa mwandishi.

Wanamtindo husisitiza umuhimu wa lugha kama chombo tekelezi kinachosaidia katika kuelewa ujumbe au maudhui yaliyokusudiwa. Nadharia hii husisitiza uwiano kati ya ujumbe na lugha na ina misingi katika dhana kwamba lugha huchangia 
ufanisi wa kazi ya sanaa kwani hutumiwa katika uzuaji na ubunifu wa kifasihi.

Nadharia hii basi hujihusisha na uchunguzi wa kinachosemwa (ujumbe) na kinavyosemwa (mtindo). Indangasi (1988) anaunga mkono maelezo ya Leech anaposema kuwa nadharia hii hujihusisha na jinsi lugha ilivyotumika kuleta mvuto kisanaa. Simpson (2004), Wamitila (2008) Leech na Short (2007) na Barry (2002) wanaainisha mihimili ya nadharia ya Mtindo ifuatavyo: lugha ya kitamathali na mtindo, uteuzi na mtindo, sarufi na mtindo, usimulizi na mtindo, chimuzi na mtindo, itikadi, mtazamo na mtindo, muwala, mshikamano na mindo na mazungumzo na mbinu za uwasilishaji, usemi na mawazo. Mihimili ya Nadharia ya Mtindo iliyotumika katika makala haya ni lugha ya kitamathali na itikadi, mtazamo na mtindo. Wamitila (2003) anasema kuwa katika ushairi aghalabu maana huwa inawasilishwa na mitindo ya kitamathali bali sio ile ya moja kwa moja. Ili kuwa na mtindo usio wa moja kwa moja kueleza maana, mwandishi anatumia tamathali chungu nzima na hususani ishara. Wanamitindo wamezingatia na kutoa nafasi kubwa kwa ishara kwani zinakuwa na ubunifu mkuu katika jamii wa kulinganisha vitu vyenye sifa wanazoelewa. Mashairi ya diwani ya Kichomi yamejaa tamathali za usemi na haswa ishara ambazo makala haya yanafasiri tofauti kulingana na matumizi yake. Matumizi ya mihimili hii katika makala haya yatasaidia kuibua ishara zilizotumika katika mashairi ya diwani ya Kichomi ili kujenga mtazamo na itikadi ya mwandishi kuhusu ubwege na hivyo basi kuibusha ishara bwege kwa sababu mwandishi anaangazia kazi yake kwa ishara mbalimbali zinazoelekeza mtazamo na itikadi.

\section{Ishara katika diwani ya Kichomi}

Euphrase Kezilahabi ni mshairi maarufu wa Kiswahili ambaye amepata kutunga mashairi aula ya kisasa. Kichomi, diwani yake Kezilahabi ya kwanza, ilichapishwa mwaka 1974, na kuweka jukwaani ushairi usiofuata kanuni za kimapokeo. Kezilahabi aliweka wazi kanuni ya sanaa ambayo ushairi wake ungezingatia: "Jambo ninalojali sana katika ushairi ni utumiaji wa picha au taswira" (Uk: xiv). Kwa kuwa picha au taswira ni pana, tasnifu hii imechunguza kipengele kimoja cha taswira: ishara. Katika sehemu hii, utafiti huu umechunguza ishara kama mbinu ya msingi anayoitumia Kezilahabi katika kuchimuza suala la ubwege katika mashairi yake. Uchanganuzi huu umehoji ishara zilizojenga mtazamo wa maisha wa Euphrase Kezilahabi na kukubaliana na mtazamo wa Gaudioso (2015) kwamba mtunzi, sawa na walivyo Camus na Nietzsche, huiangazia dunia na maisha kama yanayotamausha

Kwa msingi huu, ni dhahiri kuwa uwasilishaji ujumbe katika mashairi ya diwani ya Kichomi umefanywa kwa njia sahili ya ishara ambazo zimesheheni falsafa nzito ya maisha ambayo ni bayana tu kwa mwenye kuyatazama kwa jicho la ndani (Wamitila, 2008). Kezilahabi anaona mtindo wa lugha za ishara kama sehemu ya lugha ya kawaida ambayo imetawala mazungumzo ya binadamu wakati anapotangamana na binadamu wenzake duniani. Kwa hivyo, matumizi ya ishara za aina hii katika mashairi zimehakikiwa katika sura hii ili kubaini namna zilivyotumika katika uwasilishaji wa suala la ubwege katika ushairi huu wa kisasa. Ishara hizi zimejadiliwa ifuatavyo:.

\section{Ishara ya Mawimbi}

Mawimbi ni matuta ya maji hususan baharini na ziwani. Mshairi anatumia mawimbi kuyasawiri maisha kama bahari tesi kwenye shairi "Upepo wa Wakati" (Uk: 4). Ishara hii inaibuliwa kwenye ziwa lililosheheni mawimbi yanayocharuka kwa kupanda na kushuka, na kujisukasuka. Namna nadharia ya mtindo inavyoshikilia kwamba lugha ndicho chombo kinachosawiri maisha yalivyo, ndivyo mshairi kupitia kwa lugha ya ishara anavyoyaibua maisha yaliyojaa mawimbi yanayofifia na kuanza tena. Ishara hii ya mawimbi inawiana kwa kiasi fulani na ishara bwege zinazodhihirika kwenye shairi "Nimechoka." Namna nadharia ya mtindo inayoshikilia kwamba ni kupitia katika lugha ambapo hisia na maana ya binafsi inayomfasiri binadamu hujengwa, ndivyo ilivyo kwenye shairi "Nimechoka" ambalo linaibua hisia ya mshairi kuhusu maisha. Ishara ya maisha kuwa kichomi inaangazia maisha ya mtu binafsi yaani mshairi, ishara ya 'maisha kuwa mawimbi yanayopanda na kushuka inayasawiri maisha katika kiwango cha mtu binafsi na cha jamii. Namna ambavyo maisha ya mtu binafsi hudhihirika kuwili: kupanda na kushuka yaani mafanikio na kuanguka ndivyo pia maisha ya jamii huonyesha kupanda na kushuka.' 
Mawimbi haya yanaashiria misukosuko katika maisha. Hii ndio tasnifu ya maisha. Mathalan mtu anaweza kuwa na mafanikio makubwa kwenye kipindi fulani cha maisha yake na kisha mafanikio hayo yakatoweka katika kipindi kingine.

\section{Ishara za Mwamba ziwani na Mtu aliye Melini}

Mwamba ni jabali au jiwe kubwa linalopatikana majini au kwenye nchi kavu. Taswira ya mwamba ziwani inaibua ishara inayokinzana na ile ya mtu aliye melini kwenye shairi la "Mwamba Ziwani" (Uk: 6). Japo ishara hizi zinaashiria dhana tofauti kuhusu maisha, zinasaidia kuchimuza ubwege ambao huyasawiri maisha kama yaliyojaa misukosuko ambayo humsumbua binadamu akiwa hai hadi atakapofariki. Ishara hizi mbili: mwamba ziwani na mtu aliye melini zinakinzana na kupingana, lakini zinapambanishwa kutoa itikadi bwege ya mwandishi.

Hali hii inaoana na madai ya wanamitindo kwamba, lugha huugawa ulimwengu na kuupa maana kwa njia tofauti ambazo haziwezi kupatana ili kuunda kigezo kimoja kinachodhihirisha ukweli wa maisha. Katika shairi la "Mwamba Ziwani" (Uk:6), mshairi anauhuisha mwamba na kuufanya uzungumze. Ni dhahiri kuwa mwamba hauna uhai, umetulia na hauna kiwewe. Mawimbi yanayochimuza misukosuko kwenye maisha hayautingizi kwa vyovyote vile. Mwamba hapa unaashiria mfu ambaye haathiriwi kwa njia yoyote ile na mawimbi ya maisha. Ishara hii ya mwamba inakamilishwa na ishara ya mtu aliye melini. Meli hapa inaashiria meli ya maisha inayorakibu bahari tesi ya maisha.

Mshairi anamsawiri mtu aliye kwenye meli ziwani kama mwovu na mwingi wa wasiwasi. Isitoshe mtu huyu anasumbuliwa na misukosuko ya maisha (mawimbi) na uchu wa kila aina kiasi cha kutetemeshwa na mapaja ya msichana. Uashiriaji kwa ishara hizi unakuza suala zima la ubwege kwenye diwani teule. Dhana inayochimuzwa hapa ni kwamba mshairi hawezi kushabihiana na mwamba ziwani akiwa hai. Mshairi hawezi kuogelea (kuishi) kwenye bahari tulivu na thabiti (maisha ya amani). Mshairi akiwa angali katika meli ya maisha, bado atazidi kuandamwa na kihoro, tamaa na kupigwa na mawimbi ya maisha.

Hali hii inabua suala bwege kwamba binadamu huupata utulivu tu pindi anapofariki. Mshairi anachimuza si tu maisha yasiyokuwa na utulivu, bali yaliyosheheni kiwewe, uchu wa kila aina na mawimbi ambayo hayatulii. Kwa kuzihusisha ishara hizi mbili katika utunzi wake, mshairi anaelekea kushadidia itikadi yake kwamba maisha ni dhilifu na dhaifu, na yanayosheheni migogoro ambayo haiwezi kuisha. Ishara ya mwamba ziwani inaashiria mfu ambaye hasumbuliwi na mawimbi ya maisha. Wakati ishara ya mtu akiwa katika meli ziwani ikiibua dhana ya mtu kuhangaishwa na misukosuko aina aina. Hii ina maana kwamba, sawa na meli inavyopigwa na mawimbi na dhoruba ikiwa ziwani, ndivyo binadamu atakavyosumbuliwa na misukosuko ya maisha akiwa bado anaishi. Hali inayoibusha wazi dhana bwege kwamba kadhia za maisha ya binadamu haziwezi kupungua almradi anaishi.

\section{Ishara za Mto, Ziwa na Bahari}

Mto ni maji yaendayo wakati wote na kufanya korongo lake hususan kutoka mlimani na kuishia ziwani au baharini. Ziwa huelezea eneo kubwa la maji linalozungukwa na nchi kavu. Bahari ni mkusanyiko mkubwa wa maji ya chumvi yanayozunguka mabara ya ulimwengu na visiwa. Mpangilio wa maneno haya, "Ziwa, mto, baharimaisha" katika shairi la "Mto Nili" (Uk:7) ni muhimu kwa itikadi ya mwandishi kuhusu ubwege. Mpangilo huu wa ishara unaibua maana nyingi kuhusu maisha. Ni hali ambayo wanamitindo wanashikilia kwamba, mtindo wa lugha unaweza kuibua maana nyingi pamoja na mabadiliko yake kwa kuona maana siyo kama ilivyojengwa katika ishara zisizobadilika bali kama ilivyo katika viashiria ambapo kiashiriwa hubadilikabadilika.

Katika ushairi kama anavyosema Strang (2004), mto ni ishara ya mkondo wa maisha na bahari huashiria mwisho wa maisha ya binadamu. Kauli anayounga mkono Ferber (2007) kwamba katika fasihi, mto huashiria chanzo cha na mkondo ambao maisha ya binadamu huchukua duniani. Kwa msingi huu, ziwa linaashiria maisha ya utotoni ya mshairi ambayo ni ya amani. Tofauti na maisha ya utotoni, mkondo huu wa maisha huwa ni wa kasi kiasi kwamba binadamu hawezi kuudhibiti kwa vyovyote vile ukielekea kwa ukomo wake ambao ni kifo kinachoashiriwa na bahari. Mto Nili unaonekana kuashiria fujo za maisha bwege ya wanajamii katika dunia isiyowajali. Katika ubeti wa kwanza mshairi 
anasema kwamba anaona, '(...) dhambi zao zikielea mtoni' (Uk:7) na katika ubeti wa pili, mshairi anataja pia kwamba anaona, '(...) damu ya watu waliozama zamani ziwani’ (Uk:7).

Madondoo haya yanaashiria kwamba, kama yalivyo maji, maisha ya binadamu yamejaa matukio bwege yaliyojaa huzuni na ambayo ni mabaya zaidi na daima yanamkosesha faraja na kuyafanya maisha yake kukosa mantiki. Matukio haya yanayasawiri maisha ya binadamu kama yaliyojaa kadhia zenye dhiki. Kadhia hizi zinamwangamiza binadamu. Ni maangamizi ambayo ubwege unaishangilia kama hatua ya uhuru wa kweli. Maji ni ishara ya kioo kinachomwonyesha binadamu udhaifu na utupu wa maisha yake ambayo hana namna yoyote ya kubadili ila kuyaishi namna yalivyo. Maji haya ambayo yanaonekana kutakasa binadamu yanaoana na mantiki ya wadhanaishi kwamba Mungu hayupo na mbingu ni ombwe tupu ambalo mwanadamu alijijengea ili kukwepa ukweli wa hali yake.

\section{Ishara ya Chui-mweusi na Simba-mtu}

Wandera (2015) anadai kwamba Chui ni mnyama wa jamii ya paka ambaye ni mkali na katili. Weusi wa chui unasawiri hofu, hatari, mauti na hali isiyojulikana. Mwanga kwa mujibu wa Lawrence (2001) huwa ni ishara ya afueni na matumaini. Hall (2014) anaongezea zaidi kwa kusema kuwa mwanga huashiria pia ukweli, ufahamu, usalama au wema. Matumizi ya ishara hizi yanachangia kwa uzuaji wa suala la ubwege kwenye shairi la "Kisu Mkononi” (Uk: 13). Licha ya mtelemko (maisha) kuwa mkali (hatari), mshairi ana hiari kutelemka (kuishi). Mshairi anakiri kuwa hana hiari bali kuendelea kujiviringisha kwenye mtelemko huo mkali. Anasema kwamba mbele aendako ni sawa na chui mweusi ijapokuwa nyuma kuna mwanga.

Chui-mweusi anaashiria maisha ya alafu ya mshairi ambayo hayajui na yamejaa hatari: yaani ni chui mweusi. Mwanga wa nyuma unaashiria maisha ya mshairi nyuma ambayo anayafahamu kama yanayotamausha kwa sababu haoni chochote kizuri. Mwanga huu unampa taswira ya vifurushi vya dhambi, hali inayoibua ukweli wa maisha kama yaliyojaa fujo. Hata hivyo, mshairi hana matumaini ya kuboresha maisha yake. Hali ambayo ni dhahiri mshairi anapokiri kwamba, 'kutazama nyuma au mbele ni kufa moyo mzima.' Ishara hizi zinayamulika na kuyachimuza maisha kama yaliyosheheni ghururi, vitisho na yenye kutamausha. Licha ya maisha kuwa kadhia nyingi zinazomtamausha mshairi, anajitia ujasiri wa kuyakabili yalivyo 'kama Simba-Mtu' (Uk: 13).

Simba ni mnyama wa porini mwenye ujasiri mwingi. Ujasiri wake humfanya akabili chochote bila uoga. Kauli ambayo Wandera (2015) anaunga mkono anaposema kwamba, matumizi ya simba huwa ni ishara ya ujasiri katika jamii. Mzimu wa simba unaelekea kumsaidia mshairi ambaye anakabiliana na chui mweusi (hatari ya kuangamizwa na maisha). Simba hapa anaashiria ujasiri. Kama alivyo simba, mshairi amejitia ujasiri wa kukabiliana na ghururi za maisha bila kujali ya nyuma ambayo yanamtamausha. Kwa uamuzi huu, ishara ya simba-mtu inaibusha suala la ubwege kwamba, binadamu hana hiari ila kuyaishi maisha yalivyo.

\section{Ishara za Tabasamu na Kichomi}

Tabasamu hurejelea hali ya kucheka bila sauti. Kichomi nacho ni maumivu makali kutokana na mchomo wa kitu fulani. Sauti ya baba yake mshairi ambaye yumo miongoni mwa vichwa vyeupe ikimuasa kuendelee kuuishikilia waya: kuishi bila kukata tamaa wala kutegemea usaidizi wa yeyote hadi atakapodondoka yaani, kufa. Sauti hii inamfanya mshairi aanze kupata ung'amuzi wa nini maana ya maisha kiasi cha kuanza kutoa tabasamu (Uk: 35). Tabasamu ya mshairi imetumika kiishara. $\mathrm{Ni}$ ishara ya utambuzi wa mshairi wa maana ya maisha. Tabasamu hii inaibua kichomi ambacho kinamfanya mshairi kuyafasiri maisha kama kichomi yaani maumivu makali kutokana na kitu kinachomchoma au kinachotumika kumchomea na kumfanya atokwe na damu na kutiririkwa na machozi.

Kwa msingi huu, mahusiano kati ya kichomi na ubwege yako wazi. Binadamu anapochomwa na kichomi huhisi maumivu makali. Damu humtiririka kiasi cha kutokwa na machozi wakati mwingine. Iwapo hiki kichomi kwa mfano, ama kisu au mkuki au umambo kilimpata sehemu mbaya sana kama vile moyo au kifuani na akakosa matibabu stahiki, mtu huyo hufa. Kupitia kwa ishara hii, mshairi anaelekea kuonyesha kwamba maisha ya binadamu yamesheheni vichomi. Mshairi anayaona maisha 
kama yaliyojaa huzuni, dhiki, matatizo na mashaka yanayomuumiza binadamu, ndwele aina aina, na migongano mingi ambayo huacha alama na makovu yake kwenye umbile la mtu binafsi. Kwa msingi huu, kichomi kiubwege si silaha tu, bali ni maumivu yasababishwa na matumizi ya silaha hiyo.

Ishara ya kichomi inashirikiana na ishara zingine katika kuibusha suala zima la ubwege kwenye diwani ya Kichomi. Kwa mfano, upweke wa mshairi umesawiriwa kupitia kwa ishara kadhaa kwenye shairi la "Nimechoka." Kwanza, ipo ishara ya mtu aliyening'inia peke yake kwenye waya mgumu wa maisha. Pili, ipo ishara ya watu wanaosali bila kujali malalamiko ya mtu aliyening'inia. Tatu, ipo ishara ya mafuvu ambayo vile vile hayamjali binadamu anayening'inia. Mtu huyu kwa mujibu wa Kahigi (2020) anaonekana akiwa mpweke kabisa na ni lazima akabiliane na kichomi cha maisha peke yake, pasipo msaada. Hali hii inaoana na mtazamo wa kiubwege kwamba binadamu yu pweke na peke katika ulimwengu usiomjali na kwamba ni juu yake kuukabili bila kutarajia msaada wa yeyote.

\section{Ishara za Damu na Machozi}

Damu na machozi ni ishara za mtu anayeungulika na kuhuzunishwa na maisha. Damu hurejelea majimaji mekundu yanayozunguka kwenye mishipa ya kiumbe. Machozi nayo ni majimaji yanayotoka kwenye macho mtu akishindwa kustahimili machungu yanayotokana na dhuluma ya kuishi. Athari ya kuendelea kuushikilia waya mgumu wa maisha kwa mshairi ni dhahiri kwenye shairi la "Nimechoka" (Uk: 34). Maisha yamekwishamuumiza kwa kumkata vidole na wala hawezi kuyafuta la maumivu hayo. Kwa hivyo, maisha na tendo la kuishi yammemchosha mshairi kiasi cha sura yake kusawiri alama za uchovu. Alama hizi za uchovu zinaweza kuashiria ama alama zinazoonyesha masumbuko na mahangaiko aliyopata mshairi.

Katika shairi hili, ishara ya damu inawiana na ishara ya machozi inayojidhihiri. Binadamu anapokatwa na damu kumtoka na kumtiririka hadi kwenye kwapa kiasi cha kushindwa kuipangusa, yamkini hulia kwa kite wa yale yanayomsibu. Kwa msingi huu, machozi yanakuwa zao la kukatwa na kutiririkwa na damu. Yanaashiria machungu na huzuni iliyoko ndani ya binadamu. Huzuni ambao kiubwege unamuungulisha na kumkosesha binadamu amani.

Mshairi analia kwa kite kuomba msaada. Yamkini msaada anaoutafuta haupati. Sala inayopigwa na vichwa vyeupe ni ishara ya ubinafsi ambao umewatawala wanadamu. Kila mtu anajali nafsi yake maadam hamna wa kumsaidia. Wengi wako katika harakati zao za kusali wala hawamjali na anasalia tu kutegemea majaliwa. Hili ni dhihirisho tosha kwamba binadamu anaselea katika dunia peke yake bila yeyote wa kumjali. Sala zao ni ishara dhahiri kwamba hata Mungu wa kuwasaidia hayupo. Kuendelea kushikilia waya wa maisha unaomkata na kumroweza kwa damu ni ishara inayoibusha suala bwege kwamba binadamu ni kiumbe pweke ambaye hana yeyote wa kumwauni kutoka kwenye udhalimu wa maisha. Ubinafsi umewajaa wanadamu kiasi cha kujali tu maslahi yao duni.

\section{Ishara ya Waya Mgumu wa Maisha}

Kwa mujibu wa TUKI (2006), waya hurejelea uzi mwembamba unaotokana na chuma. Waya huu una uwezo wa kumkata na kumuumiza binadamu. Kwenye aya mwanzo ya shairi la "Nimechoka" (Kur :34-35), mshairi anaonekana amening'inizwa kwenye waya mgumu wa maisha na ambao hajui zilipofungwa ncha zao. Ishara hii ya waya mgumu wa maisha inaibua ilhamu ya kutaka kujua namna inavyochimuza suala la ubwege kwenye diwani teule. Waya mgumu unakuza vipi suala la ubwege? Kahigi (2020) anasema kwamba, 'Waya mgumu' una uwezo kukata au kuumiza, kwa msingi huu, waya mgumu unachimuza suala ubwege kwa wasifu huu wa kukata na kuumiza. Kero na matatizo ya maisha humdhulumu na kumuumiza binadamu. Isitoshe, waya mgumu unaibusha suala la ubwege kwa sababu ncha za waya hii hazionekani zilipofungwa. Kwa msingi huu, ishara ya waya mgumu inaibusha ubwege ambao chanzo na mwisho wake haujulikani

\section{Ishara za Kunaswa na Mtego wa mtoto mdogo na Vitimbo}

Kunaswa ni hali ya kushikwa kwa kitu kama vile mtego, ulimbo au gundi. Kunaswa na mtego wa mtoto mdogo kwenye shairi la "Nimechoka" (Kur:34-35), kunazidi kuibusha namna hali ya 
mshairi ilivyo bwege. Mtego wa mtoto mdogo unaashiria maisha ambayo yamemnasa mshairi wala haurejelei mtego kitu. Katika hali halisi, watoto huitega mitego yao kama sehemu ya michezo yao ya kila kuchpo, michezo ya watoto. Mshairi anaposawiri kuning'inia kwake kwenye waya kuwa sawa na hali ya ndege aliyenaswa na mtego wa mtoto mdogo, anaibua dhana kwamba maisha yamewanasa binadamu bila ufahamu wala hiari yao, sawa na namna ndege anavyonaswa kwa mtego wa mtoto mdogo bila kujua wala kuwa na hiari.

Ishara ya ndege (binadamu) kwenye mtego wa mtoto mdogo inatilia mkazo udhalimu wa maisha na hali mbovu ya mtu anayening'inia kwenye waya. Maisha yanamdhulumu na kumtesa mshairi ila hawezi kudondoka kutoka kwenye waya mgumu wa maisha licha ya mshairi kutambua wazi kuwa yeye si ndege bali ni binadamu razini. Huu ni urazini ambao unamtia hofu na kumfanya asidondoke.

Mshairi anaogopa kudondoka kutoka kwenye waya mgumu wa maisha kwa kuwa mara tu atakapodondoka, vitimbo vilivyochongwa na kuwekwa kufuata usawa wa waya vitammaliza. Ni mpangilio ambao Kahigi (2020) anashadidia kwa kusema kwamba, 'waya mgumu na vitimbo vimepangwa kwenye usawa mmoja, kimoja juu (waya) na kingine chini (vitimbo).' Ishara bia hizi zinaashiria dhana mbili tofauti: Kuning'inia kwenye waya kunaashiria hali ya kuishi na kudondoka kwenye vitimbo yaani, kufa. Waaidha, vitimbo hivi ni ishara ya hatari inayomkodolea binadamu macho. Hii ni hatari ambayo ndiyo kizingiti kikuu kwa ukombozi wake. Kupitia kwa ishara hizi, mshairi anaibusha dhana ya ubwege kwamba kuishi ni kufa. Kwa msingi huu, mshairi hana hiari ila kuendelea kushikilia waya ngumu ya maisha kwa nguvu asije akadondoka.

\section{Ishara ya Jongoo}

Wandera (2015) anasema kwamba, kitendo fulani humrudi mtu husika na kubeza sifa zake. Namna wanavyoshikilia wanamitindo kwamba maana ya kisa ni amali ya msomaji au msikizi na kwamba pana fasili mbalimbali za kisa hicho kulingana na muktadha, kisa cha Hadija kumuua mumewe (Uk :22) kinaweza kuashiria tamaa aliyonayo na inayoweza tu kumithilishwa na kisakale cha
Jongoo. Jongoo ni mdudu wa rangi nyeusi au kahawia mwenye miguu mingi sana. Mwandishi ametumia kisakale cha jongoo kutaka sana kukimbia na kumsukuma kuomba miguu elfu kisha akaachwa na nyoka, kama ishara ya tamaa aliyokuwa nayo Hadija na ambayo imemletea mkosi. Badala ya kufurahia kitendo chake, amebaki kusononeka. Hawezi kuona amani yoyote. Amebaki akihangaika tu.

\section{Ishara ya Ukucha wa Mbwa}

Mbwa ni mnyama afugwaye na binadamu ambaye hulinda na kusaidia kuwinda. Ukucha wa mbwa huwa ni sehemu ya mguu wake. Kezilahabi ameupatia ukucha huu uwezo mkubwa wa kukatiza maisha ya binadamu. Tanzia ambayo Mungah (1999) anaiona kama bwege kwa sababu inatokana na matumizi ya vifaa duni kama vile ukucha wa mbwa. Fikira kuhusu tanzia kama anavyosema Belliotti (2001), humfanya binadamu aishi maisha yenye kukata tamaa. Hali hii ni dhahiri katika shairi la "Ukucha wa Mbwa" ambapo mshairi amempa mama mjane aliyepofuka ukucha wa mbwa kumdhibiti dhidi ya wabakaji waliokuwa na mazoea ya kumbaka katika pitapita zake.

Kifaa kama hiki huashiria mazingira duni ya kuchukiza yanayomdhoofisha binadamu kimaisha na kumkosesha furaha. Ukucha huu ni zao la ushirikina ambao unadhaniwa kumkinga mama mjane baada ya Mungu kufeli kumlinda. Ukucha huu unampa matumaini ya kuishi kwa amani. Matumaini ambayo hayakudumu kwa muda mrefu. Ukucha huu wa mbwa, uliopaswa kumpa mama huyu uhuru wa kudumu dhidi ya dhuluma ya mapenzi, haukuzuia wabakaji kutekeleza unyama wao dhidi ya mama huyo, bali uliwaangamiza na vilevile kuangamiza kizazi chake chote. Ukucha wa mbwa ukawa ishara ya uhuru wa muda kwa mama mjane. Kadhalika uwezo wa ukucha wa mbwa kuua binadamu unaashiria udhaifu wa maisha ya binadamu ambayo yanaweza kukatizwa na chochote. Ukucha wa mbwa alioutafuta mama huyu unaashiria hatari ya maangamizi ya kizazi chake ambacho ulipaswa kutunza kutokana na walimwengu wenye nia mbovu. 


\section{Ishara ya Mafunza}

Mafunza ni viluwiluwi vya nzi ambavyo huonekana katika uchafu hasa vitu vinavyooza. Kwenye shairi la "Nenda Ukanywe!" (Uk: 30), mshairi anasema kwamba mafunza wamemwingia kwenye nyumba yake ya mawazo. Mafunza hapa wanaashiria mawazo kuhusu maisha ya mshairi. Wadhanaishi wanashikilia kwamba mtu anayewaza kuhusu mustakabali wa maisha yake huwa na ubwege kama sehemu ya mawazo yake. Mshairi anasema kwamba mafunza wamemuingia bongoni kuashiria kwamba mawazo juu ya maisha yake yanamshughulisha sana kiasi cha kumfanya mwehu wa kushambulia watu. Vilevile, mafunza hapa yanaweza kuashiria mahangaiko na migongano katika maisha ya binadamu. Mchezo wa mafunza unaashiria msongo wa fikira za mshairi juu ya hali yake bwege ambayo inamfanya kukosa amani na kuishia kuwashambulia wenzake. Mashambulizi haya yanaashiria maisha ya binadamu yaliyojaa fujo.

\section{Ishara ya Nzi}

Nzi ni mdudu arukaye na anapatikana mahali penye uchafu. Kezilahabi ametumia mdudu huyu katika shairi la "Nenda Ukanywe!" (Uk: 30), kuibua mandhari ya mtu anayekufa polepole. Mshairi anatumia wakati wa baridi kuashiria uzee ambao utadhibiti mchakato mzima wa kutafuta ukweli wa maisha yake. Yaani kadri wakati unavyosonga ndivyo hali yake mshairi inavyozidi kuwa mbaya. Katika ubeti huu, mshairi anaonekana kushangilia kifo chake kadri wakati unavyosonga. Mshairi anaibua ubwege wa uzeeni kupitia kwa ishara za nzi, marashi, damu na vidonda. Marashi hapa yakiashiria harufu ya mwilini. Vidonda navyo vinarejelea majeraha yanayotokana na kukatwa, kuchomwa au kudungwa na kitu. Nzi puani ni ishara ya uchafu unaotokana na kuganda kwa kamasi ambayo husababisha utata wa kupumua. Marashi, damu na vidonda vinaashiria raha, karaha na makovu aliyoyapata mshairi katika harakati za kuelewa utupu wa maisha yake.

Hali hii inamfanya mshairi kuona akikauka vile muda unavyosonga. Kukauka hapa kunaashiria kifo cha kila siku kuelekea kwa kifo halisi maana kinachokauka hakina uhai tena. Mshairi anaonekana kulemewa na maisha, hali inayoashiriwa kwa kuweka bunduki chini. Bunduki kuwa chini ni ishara tosha ya uhuru ambao ni nadra kuupata. Kwa kuwa ni kiumbe dhaifu asiyekuwa na uwezo, atakuwa kama mfungwa aliyetekwa na nguvu asizoziona. Mshairi anafahamu fika kwamba maisha ni kifo cha kila siku na ni wazi kwamba haoni tofauti kati ya maisha na kifo.

\section{Ishara ya Vichwa Vyeupe}

Vichwa vyeupe ni mafuvu ya watu waliokwisha kufa. Kwenye ubeti wa tano wa shairi "Nimechoka," mshairi anatuhamisha hadi dunia ya vichwa vyeupe au mafuvu. Haya mafuvu yanaibuka kati ya vitimbo kutoka ardhini (Uk: 35). Vichwa vyeupe vinawaashiria watu waliokwishayapita kwenye maisha na kufa; ni ishara ya mauti. Kuibuka kwa vichwa hivi kunamfanya mshairi kuyafasiri maisha kama ndoto ya uongo. Kukosa uhai kunatofautisha vichwa vyeupe na mshairi anayening'inia kwenye waya mgumu wa maisha. Kwa vile mshairi yuko hai, ana hisia na akikatwa anatokwa na damu. Ishara hii inaibusha dhana ya ubwege kwamba, binadamu atazidi kuyapata maumivu ya maisha almradi angali hai.

\section{Ishara ya vicheko vya Vichwa Vyeupe}

Vichwa hivi vinapewa sauti na uwezo wa kucheka kuashiria faraja inayotokana na kifo ambacho humrejesha binadamu katika chanzo halisi cha maisha yake. Kama anavyodai Lacey (2012), kifo ni hali nzuri ambapo kwayo binadamu hupata amani na furaha baada ya mateso ya maisha. Anavyoafiki Lacey, kifo kinampa binadamu ahadi ambayo maisha hayawezi kamwe kumpa. Vilevile vicheko vinaashiria ufahamu wa binadamu wa ukweli wa maisha yake baada ya kukumbana na mauti. Kuzuka kwa vichwa vyeupe kunamshangaza mshairi na kumfanya ahisi kuwa duni kwa kuwa haelewi uhusiano baina ya vicheko na vichwa vyeupe vilivyozuka chini yake, hali inayomfanya mshairi kukiri kwamba ukweli wa maisha ni kama ndoto ya uongo.

Vicheko vya vichwa vyeupe ni ishara ya utata na ukinzano wa maisha ya mshairi. Vichwa hivyo vinamcheka mshairi ambaye haelewi ukweli wa maisha yake. Kadhalika vichwa hivyo vinajicheka kwa kutokuwa na uwezo wa kumsaidia mshairi. Hali hii inaendeleza imani ya kiubwege kuwa binadamu ni kiumbe ambacho hakina uwezo wa 
kubadilisha hali yake wala Mungu hana lolote la kumfaa binadamu ulimwenguni. Kwa hivyo ni wazi kwamba vichwa hivi vinacheka ili kukwepa majonzi yanayotokana na hali ya kuhuzunisha, kusikitisha na kukatisha tamaa inayomkabili mshairi.

\section{Ishara ya Sauti ya Vichwa Vyeupe}

Licha ya kuwa vichwa vyeupe ni vya watu waliokwisha kufa, Kezilahabi amempatia baba yake mshairi sauti. Sauti kiuhalisia huwa ni mlio unaotokana na vitu kugusana au kugongana. Sauti vilevile hutumiwa na watu wanaoishi. Na kwa sababu sauti ni kwa aliye hai, inaashiria hisia, inayoibua ukosefu wa ukamilifu unaomfanya binadamu kuwa mwenye huzuni na dhiki, na kukosa hamu na utulivu. Sauti inaashiria upekee wa binadamu anayeishi. Tofauti na diwani ya Kichomi, msanii amempa sauti baba yake mshairi aendelee kuishi hata baada ya kufa. Kuishi huku baada ya kifo kunachimuza dhana bwege kuwa maisha si kitu muhimu bali sauti tu. Sauti anayoisikia mshairi inamfanya atambue na kuyafasiri maisha kama ndoto ya uongo. Baba anasikika akimwasa mshairi kutokata tamaa bali aendelee kuishi maisha jinsi yalivyo licha ya hali kuwa ngumu kwa kuwa itafika wakati mshairi atadondoka (kufa) (Uk : 35).

Kudondoka kunaelekea kumwondolea baba yake mshairi kichomi cha maisha. Kumempa baba yake mshairi nafasi ya kutambua na kuelewa maisha yanavyo vichomi ambavyo haviwezi kupungua hadi mauko yatakapokufika. Nasaha ya baba yake mshairi inachimuza dhana bwege kuwa juhudi za mshairi hazitamfikisha popote yamkini atadondokea (atafia) katika mazingira sawia na yale waliyofia. Kudondoka hapa kunaelekea kuashiria kifo ambacho baba yake mshairi anaona kimempa uhuru na amani ya kudumu. Hata hivyo, kifo cha mshairi ni kama ishara ya ukosefu wa uwezo wa kubadilisha hali na mkondo wa maisha yake licha ya kuzinduliwa na baba. Dhana sawa na ubwege unaoshikilia kwamba binadamu huwepo tu duniani akisubiri hatima yake asiyojua lini itatokea. Kwa hivyo, maisha hayawezi kuwa na furaha.

\section{Ishara ya Pango}

Katika diwani ya Kichomi, mtunzi anaibua mazingira ya kibunilizi yenye ishara mbalimbali za maisha. Katika diwani hii, mazingira anamojikuta binadamu si kutokana na hiari yake. Kama anavyokaulisha Reinhardt (2001:237), "binadamu hachagui hali fulani anayojikuta ndani ulimwenguni na anahisi kudhulumiwa na kuzingirwa na mazingira katili. Katika hali hii, binadamu anajihisi kama mfungwa katika ulimwengu uliomzingira." Pango lenye wavu, na mteremko mkali limetumika kuibua maisha ya binadamu yaliyojaa ghururi.

Kezilahabi anamsawiri Bikira anayeishi kwenye pango kwa takribani miaka kumi sasa akiwa amegubikwa na huzuni. Pango kiuhalisia huwa ni shimo linalopatikana chini ya ardhi, katika mti au jabali ambalo aghalabu huwa ni makazi ya wanyama mwitu. Kwa mujibu wa Magare (2002), mazingira ya pango huwa machafu na ya kihayawani. Hali ya binadamu kuishi pangoni badala ya nyumbani inaashiria maisha ya kinyama na bwege anayoishi binadamu. Sawa na mtu ambaye si hiari yake kuwa duniani, Kezilahabi amemsawiri Bikira kama kiumbe pweke na anayeishi katika mazingira duni yanayomhuzunisha na kumkosesha amani. Ni maisha ambayo yamemchosha na kumtamausha mshairi.

Mshairi na mpenzi wake hawajapata furaha katika uhusiano wao uliodumu kwa miaka kumi. Hali ya kuishi kwa huzuni inachimuza suala la ubwege kwamba maisha huzuni tupu. Kezilahabi ametumia mazingira hafifu kama pango kuyaashiria maisha duni ya binadamu. Kwa kuwa pango ni makazi ya wanyama, Kezilahabi alielekea kuashiria kwamba binadamu na maisha yake ni sawa na wanyama wa mwituni.

\section{Ishara ya Wavu}

Wavu nao hurejelea utandu wenye matundu madogomadogo ambao hutumika kama mtego. Kwenye diwani ya Kichomi (1974a:3), mshairi anasimulia miaka kumi bwege ya uhusiano wa kimapenzi na msichana aliyeishi pangoni. Maisha ya Bikira mwenye huzuni yanakuwa duni zaidi pale ambapo buibui anafuma wavu wa ubikira mlangoni. Wavu sawa na pango ni vitu vyenye uhalisia. Anavyosema Magare (2002), matumizi ya vifaa au vitu vya kiasili licha ya kuwepo vile vya kisasa kama vile nyumba na mlango ni ishara ya ubwege. Kezilahabi anapotumia wavu wa buibui badala ya mlango wa kisasa anaashiria hali ya kutobadilika 
kwa maisha ya binadamu. Binadamu anaendelea kuishi kinyama katika dunia yenye kutamausha. Kadhalika wavu huu unaashiria vizingiti anavyokumbana navyo Bikira kwenye mchakato wake wa kujitafutia uhuru utakaompa furaha na amani ya kudumu.

\section{Ishara za Gurudumu na Mtelemko Mkali}

Gurudumu ni kifaa cha mviringo kilichotengenezwa kwa mbao, chuma au mpira ambacho hutiwa kwenye chombo ili kukiwezesha kujongea (TUKI, 2004). Kadri linavyojongea ndivyo linavyozidi kuisha. Hii ndiyo fasiri ya maisha katika shairi la "Kisu Mkononi" (Uk: 13). Mtunzi anayafunua madhila na mashaka yanayosababishwa na maisha yanavyotamausha na kumfanya mshairi kutaka kujitia kitanzi kwa kisu, ijapokuwa anaghairi nia ya kujiua kwa sababu sio suluhu kwa matatizo yake. Mshairi anajiona yeye kuwa sawa na gurudumu ambalo halina budi ila kuviringika kwenye mteremko mkali huku maisha yenyewe yakiwa mteremko mkali ambao haujui mwanzo wala mwisho wake.

Ni wazi kwamba gurudumu pasipo kujua mwanzo wala mwisho, halina budi bali kutelemka hasa likiwa katika mtelemko mkali. Sawa na uashiriaji wa gurudumu, hivi ndivyo hali ya binadamu ilivyo katika maisha. Anasukumwa tu kila kuchapo. Anazidi tu kuishi huku matukio mengi yakitendeka bila hiari yake na wala hajui mwisho wenyewe wa maisha. Namna gurudumu linvyoisha kadri linavyotumika, ndivyo mshairi anavyoyaona maisha yake. Gurudumu hapa linaashiria binadamu anayezidi kufariki kadri siku na miaka inavyozidi kusonga. Binadamu hana uwezo wa kuthibiti kasi ya maisha sawa gurudumu ambalo halina uwezo juu ya kasi yake. Mtelemko unavyozidi kuwa mkali ndivyo kasi inavyozidi.

Kwa msingi huu, mtelemko (maisha) ndio unaodhibiti kasi ya maisha ya binadamu. Hivyo binadamu hana hiari ila kwenda kwa kasi na mwendo ule wa maisha japo hajui lini na wapi mwisho. Ishara ya gurudumu (binadamu) na ile ya mtelemko mkali (maisha) inachimuza dhana kwamba maisha ya binadamu yanathibitiwa na nguvu zilizo nje ya uwezo wake. Hivyo basi, uashiriaji wa ishara hizi unaibusha suala la ubwege kwamba, maisha yanathibiti mwendo na maamuzi ya mtu binafsi.

\section{Ishara ya Mti wenye Mafuriko}

Mti ni mmea wenye shina gumu unaoota mwituni au uliopandwa shambani. Mti katika jamii nyingi huwa ni ishara ya mwanamume ambaye ndiye nguzo ya familia. Kauli anayounga mkono Wandera (2015) anapodokeza kuwa mti huwakilisha mume katika boma na kukatwa kwake huacha wana wakiyumba. Hali ambayo ni dhahiri katika shairi la "Hadija" (Uk :22). Katika shairi hili, mti unaashiria mumewe Hadija, ambaye anamuua kutokana na tamaa yake ili apate uhuru wa kudumu katika ndoa yake. Kezilahabi anaonyesha kwamba uhuru alioutaka Hadija unageuka kuwa dhiki na gharika (mafuriko) inayotishia kuisambaratisha ndoa yake. Shairi hili linaashiria mwelekeo bwege wa kukatisha tamaa kuhusu maisha ya binadamu duniani. Mshairi anasema kwamba Hadija amekata mti mtima na umeangukia nyumba yake. Usemi wa 'kukata mti mtima,' unaashiria tendo la kuua, na 'kuangukia nyumba yake,' ni ishara kwamba msiba huo utamrudia yeye mwenyewe.

Kwa kuukata mti, mshairi anakariri kwamba Hadija ameziba mto hasira na nyumba yake itakuwa mafurikoni ambapo atahama na kuwakimbia watoto. Mafuriko ni maji yaliyojaa kwenye mto mpaka yakavuka kingo zake. Mwandishi ametumia ishara ya kuziba mto hasira kumaanisha kwamba amechochea msiba nyumbani kwake. Nyumba yake kuwa mafurikoni inaashiria kukumbwa na matatizo mengi. Hali anayokaulisha Diyanni (1998) anaposema kuwa, maji yanayojitokeza kama mafuriko ni ishara ya kifo au majaribu. Hii ina maana kwamba maji, yaani mafuriko ni ishara ya tukio baya. Kwa hivyo, Kezilahabi anaonyesha kwamba Hadija hataweza kukabiliana na shida zitakazomkumba hivyo basi ataghura na kuwakimbia watoto. Mshairi anamkejeli Hadija kwa kumuua mumewe kwa sababu ya tamaa kupitia kwa ishara fiche za kumlinganisha Hadija na mbuzi aliyetaka kumkimbia chui akajigeuza panya ambaye alilia kuliwa na paka. Sasa badala ya kupata uhuru alioutarajia, amekumbwa na adhabu kali zaidi ambapo mwisho wake ni kuteseka na kuangamia. 


\section{Ishara za Giza na Mwangaza}

Mifano ya ishara za hali zinazoibusha suala la ubwege kwenye mashairi ya diwani teule ni mwangaza na giza. Kimtindo, mwangaza huwa ni ishara ya ukweli, ufahamu, usalama au wema (Hall 2014), huku giza likiashiria uovu, hatari au ujinga (Frye na wenzake 1985). Giza ni hali ya kutokuwepo na nuru huku mwangaza ukirejelea uwepo wa nuru. Kezilahabi vilevile alitumia mandhari na dhana zilizosheheni ishara za giza na mwangaza. Mungah (1999) anadai kwamba mandhari yenye giza hutishia na kumtamausha binadamu anayetafuta maana ya utupu wa maisha yake. Hali ya mwangaza humsaidia kuelezea maana ya maisha yake duni duniani. Binadamu amegubikwa na giza totoro na ni sharti atafute mwangaza ili aweze kupata ufahamu wa ukweli wa uduni wa hali yake duniani.

Kezilahabi ametumia maneno giza na mwangaza katika mashairi yake kuashiria utupu wa maisha ya binadamu ambayo yamekosa mwelekeo na yanayoendelea kufikia kikomo. Katika shairi "Wanafiki, ” mshairi anasema kwamba, 'Kwa kuvaa kaniki na kuzunguka na giza usiku na mchana....Siku ya jua mwanga kutoa, nguo za giza kuwatoka,'(Uk:24). Jua hutoa miale ambayo huleta mwangaza penye giza. Jua hapa laelekea kuashiria juhudi za binadamu kutafuta ukweli na ufahamu wa maana ya uduni wa hali yake. Kadhalika jua linaelekea kuweka wazi ukweli kwamba binadamu hafahamu wala haelewi dunia anamoishi. Anadanganyika kuwa anaishi vyema na yu salama. Ameshindwa kutambua na kuona ukweli wa hadaa ya maisha anayoyaishi. Mwanga utakapotawala na ukweli kuhusu utupu wa hali yao kudhihirika wazi, binadamu watafahamu fika kwamba wao ni viumbe dhaifu wasio na ufahamu wa maisha yao yasiyokuwa na maana.

Kadhalika giza lenye mwanga linamgubika mshairi katika shairi "Tatizo." Mshairi anasema kwamba, 'Nje kuna giza lenye mwanga, $\mathrm{Na}$ ndani kuna mwanga wenye giza, Nami nimekaa pasi mwanga wala giza,'(Uk:50). Nje kuwa na giza lenye mwanga ni ishara kwamba licha ya binadamu kuwa katika hali ya hatari (giza), anaweza kuwa na matumaini ya usalama (mwanga) katika maisha. Ila matumaini haya ni kama maluweluwe kwa sababu mwanga wenyewe unaonekana kuwa wenye giza.
Mwanga huu unaashiria maisha ya mshairi ya nyuma ambayo anayafahamu kama yanayotamausha kwa sababu haoni chochote kizuri. Mwanga huu unampa taswira ya vifurushi vya dhambi, hali inayoibua ukweli wa maisha kama yaliyojaa fujo. Mwanga wenye giza unaashiria kuwa binadamu haelewi wala haoni maana ya uduni wa maisha yake ambayo ni wazi. Mshairi ametamauka na kukaa pasi mwanga wala giza. Hali ambayo kama wanavyoshikilia wanamtindo kwamba maana yake ni amali ya msomaji inaweza kufasiriwa kama ishara ya upweke na utupu wa maisha ya binadamu ambayo hana mwao wowote kuyahusu ila kuyaishi namna yalivyo.

\section{Ishara ya Vileo}

Vileo ni vinywaji ambavyo hulevya. Unywaji wa vileo mara nyingi hutumiwa kuashiria starehe na kumsahaulisha binadamu matatizo yanayomsibu katika dunia bwege inayomdhulumu kama anavyosema mimi wa shairi katika shairi la "Wimbo wa Mlevi" (Uk: 64). Mshairi mlevi ameikubali hali yake kiasi kwamba angependa kuwa yeyote mradi aruhusiwe kunywa pombe yake. Pombe inamlevya kiasi cha kusahau maisha ambayo hayathaminiki.

Katika shairi hili la "Nenda Ukanywe!" (Uk: 36), 'mimi' wa shairi anaona kifo chake kitampa utulivu na amani ya kudumu kama anavyoashiria mwandishi kupitia kwa maswali balagha na nidaa. Mimi anaonekana kuwashauri wanaomwaga machozi kwenye kaburi lake waende wakanywe ili wafurahie uduni wa hali yao. Mshairi anaonekana kufurahia kifo ambacho anaona kitampa uhuru na amani ya kudumu ambao waombolezaji hawawezi kung'amua. Kuwaamrisha waende kunywa kunaelekea kuashiria kwamba ni kileo tu kitakachowasahaulisha hali bwege wanayoselea ndani yake. Ni hali ambayo inawatesa na ambayo sasa kifo kimemwepusha. Uashiriaji huu unaibua dhana kwamba binadamu hana budi ila kushikana na maisha yalivyo akisubiri hatima yake na hivyo basi kuchangia kwa ukuzaji wa suala la ubwege katika diwani teule.

\section{Ishara ya Sahani yenye Joto}

Sahani katika hali halisi ni chombo cha plastiki au bati chenye umbo la mviringo kinachotumiwa kuandalia chakula. Joto huwa ni hali ya hewa kuwa 
moto. Dunia kuwa namna fulani ya duara (sahani) ambalo halina mwanzo wala mwisho linamfanya mshairi kukiona kitendo cha kuishi kama joto (adhabu) linalomkosesha amani. Ukosefu huu wa amani ndio unaoibusha ubwege unaomtesa mshairi. Ubwege huu ndio kina Njogu na Chimerah (1999) wanaitikadi kuwa maumivu ya kifalsafa kuhusu maisha ya binadamu yasiyokuwa na mwelekeo na yaliyotamauka. Wanasisitiza kwamba ubwege unayasawiri maisha kama adhabu kali inayomkosesha binadamu furaha.

Mateso ya maisha duniani yanamsukuma mshairi kuyamithilisha na sahani yenye joto kwenye shairi la "Mto Nili" (Uk:7). Sahani inaashiria dunia katili huku joto kali likiwa ishara ya madhila anayopitia binadamu chini ya jua ambalo humchoma katika juhudi za kutafuta maana ya maisha yake. Joto hili ndilo adhabu na kizingiti kikuu kinachomkosesha binadamu furaha na kumfanya ayaone maisha kama yaliyojaa karaha. Anakosa uhuru anaoutafuta, hali inayomsukuma mshairi kudai kwamba ni adhabu kupaswa kuishi. Hata hivyo, amani na furaha anayopata binadamu maishani haiwezi kudumu, hali inayoyafanya maisha yake kuwa ya huzuni tupu.

\section{Ishara ya kifurushi cha Pamba}

Hofu ya kudungwa na miti ilichongwa kama mishikaki inamfanya mshairi ashike waya mgumu wa maisha kwa nguvu ili asije akaanguka 'kama kifurushi cha pamba cha mtoto mdogo kilichokwisha pimwa' (Uk: 34). Katika kifungu hiki, mshairi anafananisha kuanguka (kifo) kwake na kifurushi cha pamba cha mtoto mdogo kilichokwisha pimwa. Ishara ya kifurushi cha pamba inayoibuliwa na mshairi ni muhimu katika uibushaji wa itikadi ya ubwege ya mwandishi wa diwani ya Kichomi.

Kwa kawaida, pamba hutumiwa kupangusa au kufunika vidonda na kisha hadhi yake kupotea baada ya kutumiwa. Pamba vilevile huwa nyepesi kwa uzani kiasi kwamba hata ikianguka huwezi kuhisi uzito wake. Mara nyingi watoto wanapoichezea pamba, huimega na kuiweka kwa uzani usiokadirika. Kwa msingi huu, kifurushi cha pamba cha mtoto mdogo kilichokwisha pimwa ni ishara inayoelekea kuwakilisha mshairi ambaye maisha yake hayathaminiki na muda wake duniani umeshakadiriwa (pimwa) namna pamba ilivyopimwa. Aidha, kuanguka kama kifurushi cha pamba kunaashiria kwamba kifo (kuanguka) cha mshairi hakuwezi kuutikisa ulimwengu kwa sababu hakina uzito wowote.

\section{Hitimisho}

Ishara zilizotumika kuibusha suala la ubwege kwenye diwani ya Kichomi zimetathminiwa kwa kutumia nadharia ya mtindo ya Leech (1969). Imebainika wazi kuwa, ishara zinazojitokeza kwenye diwani ya Kichomi zina muundo wa kiashirii na kiashiriwa. Ishara hizi zinaibusha binadamu aliyeshikamana na maisha kwa kuwa hana hiari. Anahisi kuchoshwa na mateso ya maisha lakini hana budi ila kuyastahimili. Huu ndio ukweli wa maisha kwani maamuzi ya waja hubadilika badilika kutegemea muktadha husika. Maisha humuumba mhusika kama ilivyobainika kupitia ishara ya Simba-mtu kwenye shairi la Kisu Mkononi. Hali hii inasadifu nadharia ya makala hii inayodai kuwa ni kwenye matumizi ya lugha tu ambapo maudhui na dhamira za kazi ya fasihi husukwa na kufafanuliwa. Kulingana na matokeo ya makala hii, utafiti huu umeweza kudadavua ishara bwege kwenye mashairi teule ya diwani ya Kichomi.

\section{Marejeleo}

Barry, P. (2002). Beginning Theory: An Introduction to Literary and Cultural Theory ( $\left.2^{\text {nd }} E d\right) . M a n c h e s t e r: M a n c h e s t e r$ University Press

Belliotti, R. (2001). What is the meaning of human life? Amsterdam: Editions Rodopi B.V.

Bennette, M. (2011). Reassessing the Theatre of the Absurd. New York: Palgrave Macmillan.

Camus, A. (1961). Resistance, Rebellion, and Death. Translated by Justin O'Brien. New York: Knopf.

Camus, A. (1961). The Outsider, Trans. (Stuart Gilbert). Hammondsworth: Penguin.

Camus, A. (1991). The Rebel: An Essay on Man in Revolt, Trans. (Anthony Bower). New York: Vintage International. 
Camus, A. (2005). The Myth of Sisyphus, Trans. (Justin O'Brien). London: Penguin.

Camus, A. (2009). The Plague, Trans. (Robin Buss). London: Penguin.

Diegner, L. (2002). Allegories In Euphrase Kezilahabi's Early Novels. AAP 72/ Swahili Forum, IX, 43-74.

Diegner, L. (2005). Intertextuality in the Contemporary Swahili Novel: Euphrase Kezilahabi's Nagona And William E. Mkufya's Ziraili na Zirani. Swahili Forum 12: 25-35.

Diyanni, R. (1998). Literature: Reading Fiction, Poetry And Essay (4th Ed). Boston: Macgraw Hill.

Edeh, A. (2019). Existential Absurdity and Alienation in Samuel Becketts's waiting for Godot and Franz's Kafka's The Metamorphosis: An Existential Analysis. Master's Thesis: University of Oulu

Esslin, M. (2001). The Theatre of the Absurd. New York: Vintage Books.

Ferber, M. (2007). A Dictionary of Literary Symbols. Cambridge: Cambridge University Pres

Frye, N., Baker, S., \& Perkins, G. (1997). The Harper Handbook Of Literature. New York: Harper Collins.

Gaudioso, R. (2014). Kuwako na Wakati: Mipaka ya Lugha kama Hatua za Falsafa katika Mashauri ya Euphrase Kezilahabi. Swahili Forum, 21, 76-103.

Gaudioso, R. (2015). Transferring And Rewriting Freedom In Euphrase Kezilahabi. Nordic Journal Of African Studies, 23(2) 63-89.

Gromov, M. (1998). Nagona And Mzingile - Novel, Tale Or Parable? AAP, 55, 73-78.

Hall, J. (2014). Dictionary Of Subjects And Symbols In Art (Revised Edition). London: Routledge.

Indangasi, H. (1988). Stylistics. Nairobi: Nairobi University Press.
Kahigi, K. (2020). Sitiari katika Kichomi: Uchambuzi wa mojawapo ya mbinu za kifasihi ya Euphrase Kezilahabi. Swahili Forum, 27, 3256

Kezilahabi E. (1974a). Kichomi. London: Heinemann Educational Books (EA) Ltd

Kezilahabi E. (2008). Dhifa. Nairobi: Vide Muwa.

Khamis, S. (2003). Fragmentation, Orality And Magic Realism In Kezilahabi's Novel Nagona. Nordic Journal Of African Studies, 12(1), 78-91.

Kipacha, A. (2017). Uchuro Katika Nathari Teule za Euphrase Kezilahabi. Swahili Forum, 24, 115

Kipacha, A. (2019). Ushairi Msemele na Ubidaa wa Euphrase Kezilahabi. Dhifa, Eastern African Literary and Cultural Studies, 5(3), 155-169.

Lacey, A. (2012). The philosophical death in the poetry of William Wordsworth and Percy Shelley.

Lanfranchi, B. (2012). Daring To Be Destructive. Euphrase Kezilahabi's Onto Criticism. Swahili Forum, 19, 72-87.

Lawrence, L. (2001). Archetypes And Symbolss. Jenks (Hayajachapishwa).

Leech, G. \& Short, M. (2007). Style in Fiction: A Linguistic Introduction to Fictional Prose: New York: Longman Limited.

Leech, G. (1969). A linguistic Guide to Modern English Poetry. Essex: Longman Group Ltd.

Magare, F.A. (2002). Uhakiki wa Tamthilia ya Amezidi kama kazi ya kiubwege.Tasnifu ya Uzamili, Chuo Kikuu cha Nairobi (Haijachapishwa).

Mezger, Sonja (2002). Roman Catholic Faith Represented in Kezilahabi's Mzingile AAP 72/ Swahili Forum IX: 75-85

Meyer, M. (2002). The Bedford Introduction to Literature. London: Bedford and Martins.

Mohamed, S.A. (2010) Nyuso za Mwanamke .Nairobi: Longman Publishers.

28 This work is licensed under a Creative Commons Attribution 4.0 International License. 
Mukhata, C. (2015). Ishara na Uashiriaji kama Nyenzo ya Mtindo, Maana na Kiwakilisha cha Itikadi katika Riwaya ya Nyuso za Mwanamke. Swahili Forum 22:104-118

Mulokozi, M. (2017). Utangulizi. Ushairi na Uhuru: Mkusanyiko wa tungo za Abdilatif Abdalla na Euphrase Kezilahabi. Napolli: University of Napoli Press. Kur 16-38

Mumme, P. (2016). The Absurd beyond Modernism. Unpublished Doctor of Philosophy Thesis: The University Of Sydney

Mungah, C. (1999). Dhana ya Maisha Katika Novella Mbili za Euphrase Kezilahabi: Nagona na Mzingile. Tasnifu ya Uzamili, Chuo Kikuu cha Nairobi (Haijachapishwa).

Njogu, K. na Chimerah, R; (1999): Ufundishaji wa Fasihi Nadharia na Mbinu. Nairobi: Jomo Kenyatta Foundation Ltd.

Osore, M. (2011). Defamiliarization In The Novels Of E. Kezilahabi And S.A. Mohamed. London: Lambert Academic Publishing.

Perrine, L. (2014). Literature: Structure, Sound, Sense. Boston: Wadsworth

Reinhardt, K.(2001). The Existential Revolt. New York. Frederick Ungar Publishing Co.

Rettova, A. (2004). Afrophone Philosophies: Possibilities and Practice. The Reflection of Philosophical Influences in Euphrase Kezilahabi's Nagona and Mzingile. Swahili forum 11:45-68

Simpson, P. (2004). Stylistics: A Resource Book for Students. London: Routledge.

Snowden, R. (2006). Freud-The Key Ideas. (Teach Yourself Series). London: Hodder Education.

Stein, A. L. (2019)"Absurdity and Metaphysical Rebellion in the Philosophies of Albert Camus and Omar Khayyam" . Undergraduate Honors Thesis Collection. 490: Butler University

Strang, V. (2004). Meaning of Water. New York: Berg Publishers
Tarantino, E. \& Caruso, C. (2009). Nonsense and Other Senses: Regulated Absurdity in Literature. London: Cambridge Scholars Publishing

TUKI. (2006). English-Swahili Dictionary ( $3^{\text {rd }}$ Ed). Dar es Salaam: Shanghai Kangshi Printing Co., Ltd.

Wamitila, K. (1998). A Philosophical Labyrinth: Tracing Two Critical Motifs in Kezilahabi's Prose Works. Swahili Forum, 5, 79-91.

Wamitila, K. (2003). Kamusi ya Fasihi: Istilahi na Nadharia. Nairobi: Focus Publishers.

Wamitila, K. (2008). Dibaji. In Kezilahabi Euphrase, 2008: Dhifa. Nairobi: Vide Muwa

Wamitila, K. (2008). Kanzi ya Fasihi: Misingi ya Uchanganuzi wa Fasihi. Nairobi: Vide-Muwa Publishers Ltd.

Wandera, S. (2015). Taswira za Kiumenke Katika Hadithi za Abanyala wa Kenya. Tasnifu ya Uzamifu, Chuo Kikuu cha Laikipia (Haijachapishwa).

29 | This work is licensed under a Creative Commons Attribution 4.0 International License. 